Pacific Journal of Mathematic 


\section{A RIEMANNIAN SPACE WITH STRICTLY POSITIVE SECTIONAL CURVATURE}

\section{Grigorios Tsagas}

Let $M_{1}$ and $M_{2}$ be two Riemannian spaces ${ }^{1}$ with Riemannian metrics $d_{1}$ and $d_{2}$ respectively whose sectional curvature is positive constant. We consider the product of the two Riemannian spaces $M_{1} \times M_{2}$, then the Riemannian space $M_{1} \times M_{2}$ has nonnegative sectional curvature with respect to the Riemannian metric $d_{1} \times d_{2}$ but not strictly positive sectional curvature.

We can construct a Riemannian metric on $M_{1} \times M_{2}$ which approaches the Riemannian metric $d_{1} \times d_{2}$ as closely as we wish and which has strictly positive sectional curvature.

Now, our results can be stated as follows. We consider two manifolds $M_{1}\left(H_{1}-E_{1}, q_{1}\right), M_{2}\left(H_{2}-E_{2}, q_{2}\right)$ such that each of them has only one chart where $H_{1}, E_{1}$ are the south hemisphere and the equator, respectively, of a $k$-dimensional sphere $(k \geqq 2)$ and $E_{2}, H_{2}$ are also the south hemisphere and the equator, respectively, of an $n$-dimensional sphere $(n \geqq 2)$, and $q_{1}, q_{2}$ are special mappings. We also consider on $M_{1}$ and $M_{2}$ particular Riemannian metrics $d_{1}, d_{2}$, respectively, with positive constant sectional curvature. We obtain a special 1-parameter family of Riemannian metrics $F(t)$ on $M_{1} \times M_{2}$ such that $F(0)=d_{1} \times d_{2}$. We have proved that $\forall P \in M_{1} \times M_{2}$ the derivative of the sectional curvature with respect to the parameter $t$ for $t=0$ and for any plane of $\left(M_{1} \times M_{2}\right)_{P}$, is strictly positive.

1. Let $M_{1}$ be a manifold which consists of one chart $\left(H_{1}-E_{1}, q_{1}\right)$, where $H_{1}, E_{1}$ are the south hemisphere and the equator, respectively, of a $k$-dimensional sphere $S_{1}^{k}(k \geqq 2)$ and the inverse mapping of $q_{1}$ is defined as follows

$$
\begin{aligned}
q_{1}^{-1}= & \left\{x^{1}=\frac{2 u_{1}}{1+u_{1}^{2}+\cdots+u_{k}^{2}}, \cdots, x^{k}=\frac{2 u_{k}}{1+u_{1}^{2}+\cdots+u_{k}^{2}},\right. \\
x^{k+1} & \left.=\frac{u_{1}^{2}+\cdots+u_{k}^{2}-1}{1+u_{1}^{2}+\cdots+u_{k}^{2}}\right\} .
\end{aligned}
$$

$q_{1}$ maps the open set $H_{1}-E_{1}$ onto the open ball $u_{1}^{2}+\cdots+u_{k}^{2}<1$. On the manifold $M_{1}$, we take the following Riemannian metric

1 A Riemannian space is a Riemannian manifold covered with one chart ([5], p. 314). 


$$
\begin{gathered}
d_{1}=d S_{1}^{2}=\left\{d_{11}=\cdots=d_{k k}=\frac{4}{\left(1+u_{1}^{2}+\cdots+u_{k}^{2}\right)^{2}},\right. \\
\left.d_{i j}=0 \text { if } i \neq j\right\},
\end{gathered}
$$

whose sectional curvature is positive constant.

Let $M_{2}$ be another manifold which consists of one chart $\left(H_{2}-E_{2}, q_{2}\right)$, where $H_{2}, E_{2}$ are the south hemisphere and the equator, respectively, of an $n$-dimensional sphere $S_{2}^{n}(n \geqq 2)$ and the inverse mapping of $q_{2}$ is defined by

$$
\begin{aligned}
q_{2}^{-1} & =\left\{x^{1}=\frac{2 u_{k+1}}{1+u_{k+1}^{2}+\cdots+u_{k+n}^{2}}, \cdots,\right. \\
x^{n} & \left.=\frac{2 u_{k+n}}{1+u_{k+1}^{2}+\cdots+u_{k+n}^{2}}, x^{n+1}=\frac{u_{k+1}^{2}+\cdots+u_{k+n}^{2}-1}{1+u_{k+1}^{2}+\cdots+u_{k+n}^{2}}\right\} .
\end{aligned}
$$

$q_{2}$ maps the open set $H_{2}-E_{2}$ onto the open ball $u_{k+1}^{2}+\cdots+u_{k+n}^{2}<0$. On the manifold $M_{2}$, we also take the following Riemannian metric

$$
\begin{aligned}
d_{2} & =d S_{2}^{2}=\left\{d_{k+1 k+1}=\cdots=d_{k+n k+n}\right. \\
& \left.=\frac{4}{\left(1+u_{k+1}^{2}+\cdots+u_{k+n}^{2}\right)^{2}}, d_{i j}=0 \text { if } i \neq j\right\},
\end{aligned}
$$

whose sectional curvature is positive constant.

Consider the product of the two manifolds $M_{1} \times M_{2}$. Then $M_{1} \times M_{2}$ is a manifold with one chart $\left\{\left(H_{1}-E_{1}\right) \times\left(H_{2}-E_{2}\right), q_{1} \times q_{2}\right\}$.

We define a 1-parameter family of Riemannian metrics on the manifold $M_{1} \times M_{2}$ defined by

$$
d S^{2}(t)=\left\{\begin{array}{c}
g_{11}=\cdots=g_{k k}=\frac{4(1+t f)}{\left(1+u_{1}^{2}+\cdots+u_{k}^{2}\right)^{2}}, \\
g_{k+1 k+1}=\cdots=g_{k+n k+n} \\
=\frac{4(1+t \varphi)}{\left(1+u_{k+1}^{2}+\cdots+u_{k+n}^{2}\right)^{2}}, g_{i j}=0 \text { if } i \neq j,
\end{array}\right.
$$

where $-b<t<b, \varphi=\varphi\left(u_{1}, \cdots, u_{k}\right), f=f\left(u_{k+1}, \cdots, u_{k+n}\right)$.

The Riemannian metric $d S^{2}(0)$ coincides with the product Riemannian metric $d S_{1}^{2} \times d S_{2}^{2}$ of the two manifolds $M_{1}$ and $M_{2}$.

2. We shall calculate the components $R_{h i j k}$ of the Riemannian curvature tensor when the index $h=1$, because the other cases are similar to these.

If $h=1$, there exist the following distinguished cases in which $R_{1 i j k}$ do not vanish identically. 


$$
\begin{aligned}
& \quad R_{1 j i j}, j=2, \cdots, k, R_{1 k+j 1 k+j}, j=1, \cdots, n, \\
& \quad R_{1 j j l}, j \neq l, j=2, \cdots, k, l=2, \cdots, k, \\
& R_{1 j j k+l}, j=2, \cdots, k, l=1, \cdots, n, \\
& \quad R_{1 k+j k+j l}, j=1, \cdots, n, l=2, \cdots, k, \\
& R_{1 i j l}, i \neq j \neq l, i=2, \cdots, k+n, j=2, \cdots, k+n, l=2, \cdots, k+n .
\end{aligned}
$$

As it is known, $R_{1 i j k}$ is given by $([12], p .18)$

$$
\begin{aligned}
R_{1 i j l}= & \frac{1}{2}\left(\frac{\partial^{2} g_{1 j}}{\partial u_{i} \partial u_{l}}+\frac{\partial^{2} g_{i l}}{\partial u_{1} \partial u_{j}}-\frac{\partial^{2} g_{i j}}{\partial u_{1} \partial u_{l}}-\frac{\partial^{2} g_{1 l}}{\partial u_{i} \partial u_{j}}\right) \\
& -g_{r s}\left(\left\{\begin{array}{c}
r \\
i j
\end{array}\right\}\left\{\begin{array}{c}
s \\
1 l
\end{array}\right\}-\left\{\begin{array}{c}
r \\
i l
\end{array}\right\}\left\{\begin{array}{c}
s \\
1 j
\end{array}\right\}\right),
\end{aligned}
$$

where $\left\{\begin{array}{c}r \\ i j\end{array}\right\},\left\{\begin{array}{c}s \\ 1 l\end{array}\right\},\left\{\begin{array}{c}r \\ i l\end{array}\right\},\left\{\begin{array}{c}s \\ 1 j\end{array}\right\}$ are the Christoffel symbols of the second kind.

From the above formula by virtue of (1.3) we obtain

$$
\begin{aligned}
& R_{1 j 1 j}=-\frac{16(1+t f)}{A^{4}}+\frac{t^{2}}{1+t \varphi} \frac{B^{2}}{A^{4}} \sum_{i=1}^{n}\left(\frac{\partial f}{\partial u_{k+i}}\right)^{2}, j=2, \cdots, k, \\
& R_{1 k+j 1 k+j}=\frac{2 t}{(A B)^{2}}\left\{A^{2} \frac{\partial^{2} \varphi}{\partial u_{1}^{2}}+2 A u_{1} \frac{\partial \varphi}{\partial u_{1}}-2 A \sum_{i=2}^{k} u_{i} \frac{\partial \varphi}{\partial u_{i}}\right. \\
& \left.+B^{2} \frac{\partial^{2} f}{\partial u_{k+j}^{2}}+2 B u_{k+j} \frac{\partial f}{\partial u_{k+j}}-2 B \sum_{i \neq j}^{n} u_{k+i} \frac{\partial f}{\partial u_{k+i}}\right\} \\
& -t^{2}\left\{\frac{\left(\frac{\partial f}{\partial u_{k+j}}\right)^{2}}{(1+t f) A^{2}}+\frac{\left(\frac{\partial \varphi}{\partial u_{1}}\right)^{2}}{(1+t \varphi) B^{2}}\right\}, j=1, \cdots, n, \\
& R_{1 j j k+l}=t^{2} \frac{\frac{\partial f}{\partial u_{k+l}} \frac{\partial \varphi}{\partial u_{1}}}{(1+t \varphi) A^{2}}, j=2, \cdots, k, l=1, \cdots, n, \\
& R_{1 k+j k+j l}=-\frac{2 t}{B^{2}}\left\{\frac{\partial^{2} \varphi}{\partial u_{1} \partial u_{l}}+\frac{2 u_{1}}{A} \frac{\partial \varphi}{\partial u_{l}}+\frac{2 u_{l}}{A} \frac{\partial \varphi}{\partial u_{1}}\right\} \\
& +t^{2} \frac{\frac{\partial \varphi}{\partial u_{1}} \frac{\partial \varphi}{\partial u_{l}}}{(1+t \varphi) B^{2}}, j=1, \cdots, n, l=2, \cdots, k \text {, } \\
& R_{1 i j l}=0, i \neq j \neq l, i=2, \cdots, k+n \text {, } \\
& j=2, \cdots, k+n, l=2, \cdots, k+n,
\end{aligned}
$$

where 


$$
A=1+u_{1}^{2}+\cdots+u_{k}^{2}, \quad B=1+u_{k+1}^{2}+\cdots+u_{k+n}^{2} .
$$

If the functions $\phi$ and $f$ are chosen such that they satisfy the systems of partial differential equations

$$
\begin{aligned}
& \frac{\partial^{2} \varphi}{\partial u_{i} \partial u_{j}}+\frac{2 u_{i}}{A} \frac{\partial \varphi}{\partial u_{j}}+\frac{2 u_{j}}{A} \frac{\partial \varphi}{\partial u_{i}}=0, \\
& i \neq j, i=1, \cdots, k, j=1, \cdots, k, \\
& \frac{\partial^{2} f}{\partial u_{h} \partial u_{l}}+\frac{2 u_{h}}{B} \frac{\partial f}{\partial u_{l}}+\frac{2 u_{l}}{B} \frac{\partial f}{\partial u_{h}}=0, \\
& \quad h \neq l, h=k+1, \cdots, k+n, l=k+1, \cdots, k+n,
\end{aligned}
$$

respectively and if $m \in[1, \cdots, k]$ and

$$
i \in[k+1, \cdots, k+n], i \neq j \in[k+1, \cdots, k+n]
$$

or if $m \in[k+1, \cdots, k+n]$ and $i \in[1, \cdots, k], i \neq j \in[1, \cdots, k]$, then we have

$$
R_{i m m j}=t^{2} \frac{\frac{\partial f}{\partial u_{i}} \frac{\partial f}{\partial u_{j}}}{(1+t f) A^{2}}, \quad \text { or } \quad R_{i m m j}=t^{2} \frac{\frac{\partial \varphi}{\partial u_{i}} \frac{\partial \varphi}{\partial u_{j}}}{(1+t \varphi) B^{2}}
$$

We consider one partial differential equation of the system (2.8), for example,

$$
\frac{\partial^{2} \varphi}{\partial u_{1} \partial u_{2}}+\frac{2 u_{1}}{A} \frac{\partial \varphi}{\partial u_{2}}+\frac{2 u_{2}}{A} \frac{\partial \varphi}{\partial u_{1}}=0
$$

or

$$
\frac{\partial^{2} \varphi}{\partial u_{1} \partial u_{2}}+\frac{\partial \log A}{\partial u_{1}} \frac{\partial \varphi}{\partial u_{2}}+\frac{\partial \log A}{\partial u_{2}} \frac{\partial \varphi}{\partial u_{1}}=0
$$

From the first of (2.7), we conclude that

$$
\frac{\partial^{2} \log A}{\partial u_{1} \partial u_{2}}=-\frac{\partial \log A}{\partial u_{1}} \frac{\partial \log A}{\partial u_{2}} .
$$

Equation (2.11), by virtue of (2.12), takes the form

$$
\begin{aligned}
& \frac{\partial^{2} \varphi}{\partial u_{1} \partial u_{2}}+\frac{\partial \log A}{\partial u_{1}} \frac{\partial \varphi}{\partial u_{2}}+\frac{\partial \log A}{\partial u_{2}} \frac{\partial \varphi}{\partial u_{1}} \\
& \quad+\frac{\partial^{2} \log A}{\partial u_{1} \partial u_{2}} \varphi+\frac{\partial \log A}{\partial u_{1}} \frac{\partial \log A}{\partial u_{2}} \varphi=0,
\end{aligned}
$$




$$
\frac{\partial}{\partial u_{1}}\left\{\frac{\partial \varphi}{\partial u_{2}}+\frac{\partial \log A}{\partial u_{2}} \varphi\right\}+\frac{\partial \log A}{\partial u_{1}}\left\{\frac{\partial \varphi}{\partial u_{2}}+\frac{\partial \log A}{\partial u_{2}} \varphi\right\}=0,
$$

from which we obtain

$$
\frac{\partial \varphi}{\partial u_{2}}+\frac{\partial \log A}{\partial u_{2}} \varphi-\frac{v}{A}=0,
$$

where $v$ is an arbitrary function of $u_{2}, \cdots, u_{k}$.

Equation (2.13) is a linear differential equation whose general solution is

$$
\varphi=\frac{1}{A}\left(z+\int v d u_{2}\right)
$$

where $z$ is an arbitrary function of $u_{1}, u_{3}, \cdots, u_{k}$.

Relation (2.14), by virtue of the first of (2.7), takes the form

$$
\varphi=\alpha \frac{\mu\left(u_{1}, u_{3}, \cdots, u_{k}\right)+\pi\left(u_{2}, \cdots, u_{k}\right)}{1+u_{1}^{2}+\cdots+u_{k}^{2}},
$$

where $z=\alpha \mu, \int v d u_{2}=\alpha \pi$ and $\alpha$ is an arbitrary real constant.

In order for the function $\varphi$ to satisfy the rest of partial differential equations of the system (2.8), as it is easily proved that it must have the form

$$
\varphi=\alpha \frac{\varphi_{1}\left(u_{1}\right)+\cdots+\varphi_{k}\left(u_{k}\right)}{1+u_{1}^{2}+\cdots+u_{k}^{2}}
$$

where $\varphi_{1}, \cdots, \varphi_{k}$ are arbitrary functions of $u_{1}, \cdots, u_{k}$, respectively.

Similarly, in order for the function $f$ to satisfy the system of partial differential equations (2.9), it must have the form

$$
f=\alpha \frac{f_{k+1}\left(u_{k+1}\right)+\cdots+f_{k+n}\left(u_{k+n}\right)}{1+u_{k+1}^{2}+\cdots+u_{k+n}^{2}},
$$

where $f_{k+1}, \cdots, f_{k+n}$ are arbitrary functions of $u_{k+1}, \cdots, u_{k+n}$, respectively.

From (2.1), (2.2), (2.4) and (2.10), we obtain

$$
\begin{gathered}
R_{1 j 1 j}(0)=-\frac{16}{A^{4}}, R_{1 j_{1 j}}^{\prime}(0)=-\frac{16 f}{A^{4}}, j=2, \cdots, k, \\
R_{1 k+j 1 k+j}(0)=0, R_{1 k+j 1 k+j}^{\prime}(0)=\frac{2}{(A B)^{2}}\left\{A^{2} \frac{\partial^{2} \varphi}{\partial u_{1}^{2}}+2 A u_{1} \frac{\partial \varphi}{\partial u_{1}}\right. \\
\left.-2 A \sum_{i=2}^{k} u_{i} \frac{\partial \varphi}{\partial u_{i}}+B^{2} \frac{\partial^{2} f}{\partial u_{k+j}^{2}}+2 B u_{k+j} \frac{\partial f}{\partial u_{k+j}}-2 B \sum_{i \neq j}^{n} u_{k+i} \frac{\partial f}{\partial u_{k+i}}\right\}, \\
j=1, \cdots, n
\end{gathered}
$$




$$
\begin{aligned}
R_{1 j j k+l}(0) & =R_{1 j j k+l}^{\prime}(0)=0, \quad j=2, \cdots, l=1, \cdots, n, \\
R_{1 k+j k+j l}(0) & =R_{1 k+j k+j l}^{\prime}(0)=0, \quad j=1, \cdots, n, l=1, \cdots, n,
\end{aligned}
$$

where $R_{h i j l}^{\prime}$ denotes the derivative of $R_{h i j l}$ with respect to the parameter $t$.

From (1.1), (1.2) and (1.3), we obtain the following formulas

$$
\left\{\begin{aligned}
g_{11}(0)=\cdots=g_{k k}(0) & =d_{11}, \\
g_{k+1 k+1}(0)=\cdots & =g_{k+n k+n}(0)=d_{k+n k+n}, \\
g_{11}^{\prime}(0)=\cdots=g_{k k}^{\prime}(0) & =f d_{11}, \\
g_{k+1 k+1}^{\prime}(0)=\cdots & =g_{k+n k+n}^{\prime}(0)=\varphi d_{k+n k+n} .
\end{aligned}\right.
$$

Relations (2.18) and (2.19) by means of (2.7) and (2.22) take the form

$$
\begin{array}{r}
R_{1 j 1 j}=-d_{11}^{2}, R_{1 j 1 j}^{\prime}(0)=-f d_{11}^{2}, \quad j=2, \cdots, k, \\
R_{1 k+j 1 k+j}(0)=0, R_{1 k+j 1 k+j}^{\prime}(0)=\frac{d_{11} d_{k+1 k+1}}{8}\left\{A^{2} \frac{\partial^{2} \varphi}{\partial u_{1}^{2}}+2 A u_{1} \frac{\partial \varphi}{\partial u_{1}}\right. \\
\left.-2 A \sum_{i=2}^{k} u_{i} \frac{\partial \varphi}{\partial u_{i}}+B^{2} \frac{\partial^{2} f}{\partial u_{k+j}^{2}}+2 B u_{k+j} \frac{\partial f}{\partial u_{k+j}}-2 B \sum_{i \neq j}^{n} u_{k+j} \frac{\partial f}{\partial u_{k+i}}\right\} \\
j=1, \cdots, k .
\end{array}
$$

3. Let $P$ be any point of $M_{1} \times M_{2}$. Then the $k+n$ vectors $\partial / \partial u_{1}, \cdots, \partial / \partial u_{k}, \partial / \partial u_{k+1}, \cdots, \partial / \partial u_{k+n}$ form an orthonormal basis of the tangent space $\left(M_{1} \times M_{2}\right)_{P}$.

As it is known, the sectional curvature of the plane spanned by $\partial / \partial u_{1}, \partial / \partial u_{j}, j=2, \cdots, k$, is given by

$$
K_{1 j}=-\frac{R_{1 j_{1 j}}}{g_{11} g_{j j}}, \quad j=2, \cdots, k,
$$

which implies

$$
K_{1 j}^{\prime}(0)=-\frac{R_{1 j j}^{\prime}(0) g_{11}(0) g_{j j}(0)-R_{1 j 1 j}(0)\left\{g_{11}^{\prime}(0) g_{j j}(0)+g_{11}(0) g_{j j}^{\prime}(0)\right\}}{g_{11}^{2}(0) g_{j j}^{2}(0)}
$$

Relation (3.1), by virtue of (2.22) and (2.23), takes the form

$$
K_{1 j}^{\prime}(0)=-f .
$$

Similarly, calculating $K_{k+1 k+j}^{\prime}(0)$, we obtain

$$
K_{k+1 k+j}^{\prime}(0)=-\varphi \text {. }
$$

Formulas (3.2) and (3.3), by means of (2.16) and (2.17), take the form 


$$
\begin{aligned}
& K_{1 j}^{\prime}(0)=-\alpha \frac{f_{k+1}\left(u_{k+1}\right)+\cdots+f_{k+n}\left(u_{k+n}\right)}{1+u_{k+1}^{2}+\cdots+u_{k+n}^{2}}, \\
& K_{k+1 k+j}^{\prime}(0)=-\alpha \frac{\varphi_{1}\left(u_{1}\right)+\cdots+\varphi_{k}\left(u_{k}\right)}{1+u_{1}^{2}+\cdots+u_{k}^{2}},
\end{aligned}
$$

respectively. In order for $K_{i j}^{\prime}(0), K_{k+1 k+j}^{\prime}(0)$ to be positive, we must have $\alpha<0, f_{k+j}\left(u_{k+j}\right)>0, j=1, \cdots, n, \varphi_{i}\left(u_{i}\right)>0, i=1, \cdots, k$, which means the real number $\alpha$ must be negative and the functions $f_{k+j}\left(u_{k+j}\right)$ and $\varphi_{i}\left(u_{i}\right)$ must be positive when the corresponding variable takes values in the interval $(-1,1)$.

The sectional curvature of the plane spanned by $\partial / \partial u_{l}, \partial / \partial u_{k+j}$ is given by

$$
K_{l k+j}=-\frac{R_{l k+j l k+j}}{g_{l l} g_{k+j k+j}}, \quad l=1, \cdots, k, j=1, \cdots, n,
$$

which, by virtue of (2.22) and either (2.24) or similar to (2.24), takes the form

$$
\begin{aligned}
K_{l k+j}^{\prime}(0)= & -\frac{1}{8}\left\{A^{2} \frac{\partial^{2} \varphi}{\partial u_{l}^{2}}+2 A u_{l} \frac{\partial \varphi}{\partial u_{l}}-2 A \sum_{i \neq l}^{k} u_{i} \frac{\partial \varphi}{\partial u_{i}}\right. \\
& \left.+B^{2} \frac{\partial f^{2}}{\partial u_{k+j}^{2}}+2 B u_{k+j} \frac{\partial f}{\partial u_{k+j}}-2 B \sum_{i \neq j}^{n} u_{k+i} \frac{\partial f}{\partial u_{k+i}}\right\} .
\end{aligned}
$$

In order for $K_{l k+j}^{\prime}(0)$ to be positive and because the functions $\varphi$ and $f$ are independent, it must be

$$
\begin{gathered}
A^{2} \frac{\partial^{2} \varphi}{\partial u_{l}^{2}}+2 A u_{l} \frac{\partial \varphi}{\partial u_{l}}-2 A \sum_{i \neq l}^{k} u_{i} \frac{\partial \varphi}{\partial u_{i}}<0, \quad l=1, \cdots, k, \\
B^{2} \frac{\partial^{2} f}{\partial u_{k+j}^{2}}+2 B u_{k+j} \frac{\partial f}{\partial u_{k+j}}-2 B \sum_{i \neq j}^{n} u_{k+i} \frac{\partial f}{\partial u_{k+i}}<0, \\
j=1, \cdots, n .
\end{gathered}
$$

Inequalities (3.5) and (3.6), by virtue of (2.16) and (2.17), become

$$
\begin{array}{r}
\frac{\alpha}{A}\left\{A^{2} \frac{d^{2} \varphi_{l}}{d u_{l}^{2}}-2 A \sum_{i=1}^{k} u_{i} \frac{d \varphi_{i}}{d u_{i}}-2(2-A) \sum_{i=1}^{k} \varphi_{i}\right\}<0, \quad l=1, \cdots, k, \\
\frac{\alpha}{B}\left\{B^{2} \frac{d^{2} \dot{f}_{k+j}}{d u_{k+j}^{2}}-2 B \sum_{i=1}^{n} u_{k+i} \frac{d f_{k+i}}{d u_{k+i}}-2(2-B) \sum_{i=1}^{n} f_{k+i}\right\}<0, \\
\quad j=1, \cdots, n,
\end{array}
$$

which imply 


$$
\left\{\begin{array}{r}
A^{2} \frac{d^{2} \varphi_{l}}{d u_{l}^{2}}-2 A \sum_{i=1}^{k} u_{i} \frac{d \varphi_{i}}{d u_{i}}-2(2-A) \sum_{i=1}^{k} \varphi_{i}>0, \quad l=1, \cdots, k, \\
B^{2} \frac{d^{2} f_{k+j}}{d u_{k+j}^{2}}-2 B \sum_{i=1}^{n} u_{k+i} \frac{d f_{k+i}}{d u_{k+i}}-2(2-B) \sum_{i=1}^{n} f_{k+i}>0, \\
j=1, \cdots, n .
\end{array}\right.
$$

If the functions $f_{k+j}=f_{k+j}\left(u_{k+j}\right), \varphi_{i}=\varphi_{i}\left(u_{i}\right)$ are chosen to have the form

$$
f_{k+j}=u_{k+j}^{2}+\frac{1}{2 n}, j=1, \cdots, n, \varphi_{i}=u_{i}^{2}+\frac{1}{2 k}, i=1, \cdots, k,
$$

then the inequalities (3.7) take the form

$$
2-A>0, \quad 2-B>0,
$$

which, by virtue of (2.7), become

$$
1-u_{1}^{2}-\cdots-u_{k}^{2}>0, \quad 1-u_{k+1}^{2}-\cdots-u_{k+n}^{2}>0,
$$

which are valid on the open balls $u_{1}^{2}+\cdots+u_{k}^{2}<1, u_{k r 1}^{2}+\cdots+$ $u_{k+n}^{2}<1$, respectively.

Relations (2.16) and (2.17), by means of (3.8), take the form

$$
f=\alpha \frac{u_{k+1}^{2}+\cdots+u_{k+n}^{2}+1 / 2}{u_{k+1}^{2}+\cdots+u_{k+n}^{2}+1}, \quad \varphi=\alpha \frac{u_{1}^{2}+\cdots+u_{k}^{2}+1 / 2}{u_{1}^{2}+\cdots+u_{k}^{2}+1} .
$$

The second of (2.24) or similar to that and (3.4), by means of (3.9), become

$$
\begin{aligned}
R_{l k+j l k+j}^{\prime}(0)= & \frac{2 \alpha}{\left(1+u_{1}^{2}+\cdots+u_{k}^{2}\right)^{2}\left(1+u_{k+1}^{2}+\cdots+u_{k+n}^{2}\right)^{2}} \\
& \times\left\{\frac{1-u_{1}^{2}-\cdots-u_{k}^{2}}{1+u_{1}^{2}+\cdots+u_{k}^{2}}+\frac{1-u_{k+1}^{2}-\cdots-u_{k+n}^{2}}{1+u_{k+1}^{2}+\cdots+u_{k+n}^{2}}\right\}, \\
K_{l k+j}^{\prime}(0)=- & \frac{\alpha}{8}\left\{\frac{1-u_{1}^{2}-\cdots-u_{k}^{2}}{1+u_{1}^{2}+\cdots+u_{k}^{2}}+\frac{1-u_{k+1}^{2}-\cdots-u_{k+n}^{2}}{1+u_{k+1}^{2}+\cdots+u_{k+n}^{2}}\right\}, \\
& l=1, \cdots, k, j=1, \cdots, n .
\end{aligned}
$$

Using the fact that $\alpha<0$, then following inequalities are obtained from the above relations:

$$
R_{l k+j l k+j}^{\prime}(0)<0, \quad K_{l k+j}^{\prime}(0)>0, \quad l=1, \cdots, k, j=1, \cdots, n,
$$

which are valid on the open balls $u_{1}^{2}+\cdots+u_{k}^{2}<1, u_{k+1}^{2}+\cdots+$ $u_{k+n}^{2}<1$.

Let $\xi\left(\xi^{1}, \cdots, \xi^{k+n}\right)$ and $z\left(z^{1}, \cdots, z^{k+n}\right)$ be any two vectors of the tangent space $\left(M_{1} \times M_{2}\right)_{P}$. The sectional curvature of the plane spanned by $\xi$ and $z$ is given by ([11], p. 12) 


$$
K=\frac{R_{h i j l} z^{h} z^{j} \xi^{i} \xi^{l}}{\left(g_{h l} g_{i j}-g_{h j} g_{i l}\right) z^{h} z^{j} \xi^{i} \xi^{l}},
$$

or

$$
K=\frac{A_{1}}{B_{1}}
$$

where

$$
A_{1}=R_{h i j l} z^{h} z^{j} \xi^{i} \xi^{l}, \quad B_{1}=\left(g_{h l} g_{i j}-g_{h j} g_{i l}\right) z^{h} z^{j} \xi^{i} \xi^{l} .
$$

From (3.11), the following is obtained:

$$
K^{\prime}(0)=\frac{A_{1}^{\prime}(0) B_{1}(0)-A_{1}(0) B_{1}^{\prime}(0)}{B_{1}^{2}(0)} .
$$

From (3.12), by virtue of (2.3), (2.6), (2.20), (2.21), (2.22), (2.23), (2.24) and similar formulas to $(2.23)$ and $(2.24)$, we obtain

$$
\begin{gathered}
A_{1}(0)=-C d_{11}^{2}-D d_{k+1 k+1}^{2}, \\
A_{1}^{\prime}(0)=-f C d_{11}^{2}-\varphi D d_{k+1 k+1}^{2}+T, \\
B_{1}(0)=-C d_{11}^{2}-D d_{k+1 k+1}^{2}-E d_{11} d_{k+1 k+1}, \\
B_{1}^{\prime}(0)=-2 f C d_{11}^{2}-2 \varphi D d_{k+1 k+1}^{2}-(f+\varphi) E d_{11} d_{k+1 k+1},
\end{gathered}
$$

where

$$
\begin{gathered}
C=\sum_{i=1}^{k} \sum_{i<j=2}^{k} \alpha_{i j}^{2}, \quad D=\sum_{i=k+1}^{k+n} \sum_{i<j=k+2}^{k+n} \alpha_{i j}^{2}, \quad E=\sum_{i=1}^{k} \sum_{j=1}^{n} \alpha_{i k+j}^{2}, \\
T=\sum_{l=1}^{k} \sum_{j=1}^{n} R_{l k+j l k+j}^{\prime}(0) \alpha_{l k+j}^{2}, \alpha_{j m}=\left(z^{i} \xi^{m}-z^{m} \xi^{i}\right) .
\end{gathered}
$$

Relation (3.13), by means of (3.14), takes the form

$$
K^{\prime}(0)=\frac{T B_{1}(0)+C G d_{11}^{2}+D J d_{k+1 k+1}^{2}}{B_{1}^{2}(0)},
$$

where

$$
G=B_{1}^{\prime}(0)-f B_{1}(0), \quad J=B_{1}^{\prime}(0)-\varphi B_{1}(0) .
$$

Formulas (3.20), by virtue of (3.15), and (3.16), become

$$
G=L-(2 \varphi-f) D d_{k+1 k+1}^{2}, \quad J=N-(2 f-\varphi) C d_{11}^{2},
$$

where

$$
\begin{aligned}
& L=-\varphi E d_{11} d_{k+1 k+1}-f C d_{11}^{2}, \\
& N=-f E d_{11} d_{k+1 k+1}-\varphi D d_{k+1 k+1}^{2}
\end{aligned}
$$


Relation (3.19), by means of (3.21), takes the form

$$
K^{\prime}(0)=\frac{T B_{1}(0)+C L d_{11}^{2}+D N d_{k+1 k+1}^{2}-(f+\varphi) C D d_{11}^{2} d_{k+1 k+1}^{2}}{B_{1}^{2}(0)} .
$$

From (3.15) and (3.22), by means of (3.17), and because the functions $f$ and $\varphi$ are negative, we conclude

$$
B_{1}(0)<0, \quad L \geqslant 0, \quad N \geqslant 0 \text {. }
$$

The first of (3.18), by virtue of the first inequality of (3.10), implies

$$
T \leqq 0
$$

Formula (3.23), by means of (3.17), (3.24), (3.25) and $f<0, \varphi<0$, implies

$$
K^{\prime}(0)>0,
$$

because it is not possible that simultaneously $C=D=T=0$ for the two vectors $\xi$ and $z$.

Hence, we have the following theorem.

THEOREM. Let $M_{1}$ and $M_{2}$ be two special Riemannian spaces with constant positive sectional curvature defined in $\S 1$. If we consider a special 1-parameter family of Riemannian metrics $F(t)$ on $M_{1} \times M_{2}$ defined by (1.3), where the functions $f, \phi$ have the form (3.9), then the derivative of the sectional curvature with respect to the parameter $t$ for $t=0$ and for any plane of $\left(M_{1} \times M_{2}\right)_{P}$ and $\forall P \in M_{1} \times M_{2}$ is strictly positive.

From the above, we conclude that, if the parameter $t$ is positive and small enough, then the corresponding Riemannian metric $F(t)$ defined by (1.3) on $M_{1} \times M_{2}$, where the functions $f$ and $\varphi$ have the form (3.9), has strictly positive sectional curvature.

I wish to express here my thanks to Professor S. Kobayashi for many good ideas I obtained from conversations with him.

\section{REFERENCES}

1. M. Berger, Trois remarques sur les variétiés riemanniennes à courbure positive,

C. R. Acad. Sc. Paris 263 (1966), 76-78.

2. R. Bishop and R. Crittenden, Geometry of Manifolds, Academic Press, New York, 1964.

3. L. Eisenhart, Riemannian Geometry, Princeton University Press, 1949.

4. T. Frankel, Manifolds with positive curvature, Pacific J. Math. 11 (1961), 165-174. 
5. H. Guggenheimer, Differential Geometry, McGraw-Hill Book Company, 1963.

6. N. Hicks, Notes on Differential Geometry, Math. Studies No. 3, Van Nostrand, New York, 1965.

7. S. Kobayashi and K. Namizu, Foundations of Differential Geometry, Vol. 1, Interscience, New York, 1963.

8. S. Myers, Riemannian manifolds with positive mean curvature, Duke Math. J. 8 (1941), 401-404.

9. S. Sternberg, Lectures on Differential Geometry, Prentice-Hall, Englewood Cliffs, N. J., 1964.

10. Y. Tsukamoto, On Riemannian manifolds with positive curvature, Mem. Fac. Sci. Kyushu Univ. 15 (1961), 90-96.

11. K. Yano, Differential geometry on complex and almost complex spaces, Pergamon Press, New York, 1965.

12. K. Yano and S. Bochner, Curvature and Betti numbers, Ann. of Mat. Stud. 32, Princeton University Press, 1953.

Received March 14, 1967. 



\section{PACIFIC JOURNAL OF MATHEMATICS}

\section{EDITORS}

\section{H. ROYDEN}

Stanford University

Stanford, California
J. DugundJI

Department of Mathematics

Rice University

Houston, Texas 77001

RICHARD ARENS

University of California

Los Angeles, California 90024

Seattle, Washington 98105

\section{ASSOCIATE EDITORS}
E. F. BeCKENBACH
B. H. NeumanN
F. WOLF
K. YOSIDA

\section{SUPPORTING INSTITUTIONS}

\author{
UNIVERSITY OF BRITISH COLUMBIA \\ CALIFORNIA INSTITUTE OF TECHNOLOGY \\ UNIVERSITY OF CALIFORNIA \\ MONTANA STATE UNIVERSITY \\ UNIVERSITY OF NEVADA \\ NEW MEXICO STATE UNIVERSITY \\ OREGON STATE UNIVERSITY \\ UNIVERSITY OF OREGON \\ OSAKA UNIVERSITY \\ UNIVERSITY OF SOUTHERN CALIFORNIA
}

\author{
STANFORD UNIVERSITY \\ UNIVERSITY OF TOKYO \\ UNIVERSITY OF UTAH \\ WASHINGTON STATE UNIVERSITY \\ UNIVERSITY OF WASHINGTON \\ * * * * \\ AMERICAN MATHEMATICAL SOCIETY \\ CHEVRON RESEARCH CORPORATION \\ TRW SYSTEMS \\ NAVAL WEAPONS CENTER
}

Mathematical papers intended for publication in the Pacific Journal of Mathematics should be in typed form or offset-reproduced, double spaced with large margins. Underline Greek letters in red, German in green, and script in blue. The first paragraph or two must be capable of being used separately as a synopsis of the entire paper. It should not contain references to the bibliography. Manuscripts, in duplicate if possible, may be sent to any one of the four editors. All other communications to the editors should be addressed to the managing editor, Richard Arens, University of California, Los Angeles, California 90024.

Each author of each article receives 50 reprints free of charge; additional copies may be obtained at cost in multiples of 50 .

The Pacific Journal of Mathematics is published monthly. Effective with Volume 16 the price per volume (3 numbers) is $\$ 8.00$; single issues, $\$ 3.00$. Special price for current issues to individual faculty members of supporting institutions and to individual members of the American Mathematical Society: $\$ 4.00$ per volume; single issues $\$ 1.50$. Back numbers are available.

Subscriptions, orders for back numbers, and changes of address should be sent to Pacific Journal of Mathematics, 103 Highland Boulevard, Berkeley 8, California.

Printed at Kokusai Bunken Insatsusha (International Academic Printing Co., Ltd.), 7-17, Fujimi 2-chome, Chiyoda-ku, Tokyo, Japan.

PUBLISHED BY PACIFIC JOURNAL OF MATHEMATICS, A NON-PROFIT CORPORATION

The Supporting Institutions listed above contribute to the cost of publication of this Journal, but they are not owners of publishers and have no responsibility for its content or policies. 


\section{Pacific Journal of Mathematics \\ Vol. 25, No. $2 \quad$ October, 1968}

Martin Aigner, On the tetrahedral graph ..................... 219

Gregory Frank Bachelis, Homomorphisms of annihilator Banach

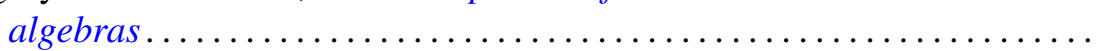

Phillip Alan Griffith, Transitive and fully transitive primary abelian groups.......................................... 249

Benjamin Rigler Halpern, Fixed points for iterates . . . . . . . . . . . . . 255

James Edgar Keesling, Mappings and dimension in general metric spaces ......................................... 277

$\mathrm{Al}$ (Allen Frederick) Kelley, Jr., Invariance for linear systems of ordinary differential equations ................................ 289

Hayri Korezlioglu, Reproducing kernels in separable Hilbert spaces . . . . . 305

Gerson Louis Levin and Wolmer Vasconcelos, Homological dimensions and Macaulay rings ................................. 315

Leo Sario and Mitsuru Nakai, Point norms in the construction of harmonic

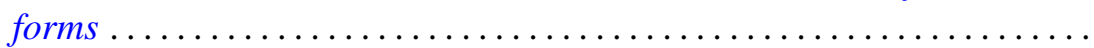

Barbara Osofsky, Noncommutative rings whose cyclic modules have cyclic injective hulls ..................................... 331

Newton Tenney Peck, Extreme points and dimension theory........... 341

Jack Segal, Quasi dimension type. II. Types in 1-dimensional spaces ...... 353

Michael Schilder, Expected values of functionals with respect to the Ito distribution ...

Grigorios Tsagas, A Riemannian space with strictly positive sectional curvature

John Alexander Williamson, Random walks and Riesz kernels . . 